\title{
BMJ Open Effects of switching from a dipeptidyl peptidase- 4 inhibitor to luseogliflozin on nocturnal blood pressure in patients with type 2 diabetes: protocol for a multicentre, prospective, randomised, open-label, blinded endpoint parallel- group comparison study
}

\author{
Reina Kameda, ${ }^{1}$ Hiroshi Nomoto, ${ }^{1}$ Kyu Yong Cho, ${ }^{1,2}$ Shinichiro Kawata, ${ }^{1}$ \\ Kazuno Omori, ${ }^{1}$ Jun Takeuchi, ${ }^{3}$ So Nagai, ${ }^{4}$ Yoshio Kurihara, ${ }^{5}$ Shin Aoki, ${ }^{6}$ \\ Akinobu Nakamura, ${ }^{1}$ Tatsuya Atsumi, ${ }^{1}$ Hideaki Miyoshi (i] ${ }^{7}$
}

To cite: Kameda R, Nomoto $\mathrm{H}$, Cho KY, et al. Effects of switching from a dipeptidyl peptidase-4 inhibitor to luseogliflozin on nocturnal blood pressure in patients with type 2 diabetes: protocol for a multicentre, prospective, randomised, open-label, blinded endpoint parallel-group comparison study. BMJ Open 2020;10:e034883. doi:10.1136/ bmjopen-2019-034883

- Prepublication history for this paper is available online. To view these files, please visit the journal online (http://dx.doi org/10.1136/bmjopen-2019034883).

RK and HN contributed equally.

Received 09 0ctober 2019 Revised 09 January 2020 Accepted 09 January 2020

Check for updates

(c) Author(s) (or their employer(s)) 2020. Re-use permitted under CC BY-NC. No commercial re-use. See rights and permissions. Published by BMJ.

For numbered affiliations see end of article.

Correspondence to Dr Hideaki Miyoshi; hmiyoshi@med.hokudai.ac.jp

\section{ABSTRACT}

Introduction Nocturnal hypertension is clinically important for patients with type 2 diabetes (T2D), considering its strong correlation with cardiovascular events. We aim to test the hypothesis that the sodiumglucose cotransporter 2 inhibitor, luseogliflozin, ameliorates nocturnal hypertension more effectively than a dipeptidyl peptidase (DPP)-4 inhibitor in patients with T2D. Methods and analysis This study is a multicentre, prospective, randomised, open-label, blinded endpoint parallel-group trial. Sixty participants with T2D and hypertension who have been treated with a DPP-4 inhibitor for more than 4 weeks and who have a glycated haemoglobin $\mathrm{A} 1 \mathrm{c}(\mathrm{HbA1c})$ level of $6.0 \%-9.0 \%$ will be randomised based on age, body mass index (BMI) and $\mathrm{HbA} 1 \mathrm{C}$ to continue taking their DPP-4 inhibitor or to switch to luseogliflozin $2.5 \mathrm{mg}$ once daily for 8 weeks. Twentyfour-hour ambulatory blood pressure monitoring (ABPM) will be performed twice at baseline and at the end of the study. All participants will continue their diet and exercise therapy, and the doses of concomitant medications will not be adjusted during the study. The primary endpoint is the effect of luseogliflozin on the mean change in systolic blood pressure (SBP) during the night, as measured by ABPM. The secondary endpoints are mean change in diastolic blood pressure (DBP) during the night, 24 hours of SBP and DBP, daytime SBP and DBP, pulse rate, BP $M$-value, trough SBP and DBP for 1 hour before the next dose, and other laboratory parameters. The sample size was calculated for a two-sided test at $90 \%$ power for the detection of a difference between treatments.

Ethics and dissemination The Ethics Review Board of Hokkaido University Hospital has approved the protocol. The results will be disseminated in peer-reviewed journals and at scientific conferences

Trial registration numbers The University Hospital Medical Information Network (UMIN000031451); Japan Registry of Clinical Trials (jRCTs011180019); Pre-results.
Strengths and limitations of this study

This randomised controlled study will be the first to directly compare the nocturnal anti-hypertensive efficacy of luseogliflozin and dipeptidyl peptidase (DPP)-4 inhibitors in patients with type 2 diabetes, using ambulatory blood pressure monitoring.

- Since DPP-4 inhibitors are the most frequently used anti-diabetic drugs in Japan, it is important to know whether switching from DPP-4 inhibitors to luseogliflozin will have merit on managing nocturnal hypertension.

- This study will be conducted in a standard clinical practice setting with broad eligibility criteria, which reflects real world.

- Participants are not blinded to their treatment although this study is a multicentre, prospective, randomised, blinded endpoint parallel-group trial.

\section{INTRODUCTION}

The prevention and management of atherosclerotic diseases, such as cardiovascular disease (CVD) and stroke, is a key issue in the management of patients with type 2 diabetes (T2D). ${ }^{1}$ It is well known that appropriate control of hypertension in patients with T2D reduces their risk of developing CVD. ${ }^{23}$ Therefore, monitoring blood pressure (BP) is as important as glycaemic control in this population. It has recently been established that not only clinic BP, but also 24-hour BP, including that during the night and morning, are important risk factors for CVD. In fact, diabetic patients who show nocturnal high BP, called 'non-dipper' and/or 'riser' types, 
are now proven to be at high risk of cardiovascular mortality. ${ }^{45}$

Sodium-glucose cotransporter 2 (SGLT2) inhibitors are drugs that reduce blood glucose by increasing kidney function-dependent and blood glucose-dependent urinary glucose excretion. ${ }^{6}$ These agents have been shown not only to have a hypoglycemic effect, but also extra-glycemic effects, including body weight and BP reduction. In addition, there is accumulating evidence that these drugs reduce the incidence of cardiovascular events in diabetic populations. ${ }^{7-9}$ A meta-analysis of 27 randomised controlled trials revealed that SGLT2 inhibitors significantly reduce both systolic $\mathrm{BP}$ and diastolic BP (by $4.0 \mathrm{~mm} \mathrm{Hg}$ and $1.6 \mathrm{~mm} \mathrm{Hg}$, respectively) from baseline. ${ }^{10}$ Incretin drugs, including dipeptidyl peptidase-4 (DPP-4) inhibitors, are also known to have modest BP-lowering effects. ${ }^{11}$ However, the effects of switching from a DPP-4 inhibitor to an SGLT2 inhibitor on BP have not been studied to date, despite such a switch being frequently made in clinical practice.

Therefore, in this prospective, randomised, open-label, parallel-group trial, we will compare the effects of these agents on BP and heart rate in T2D patients with hypertension, using ambulatory blood pressure monitoring (ABPM), in order to evaluate nocturnal and morning BP.

\section{METHODS}

\section{Study design}

This is a multicentre, open-label prospective, randomised, blinded endpoint parallel-group comparison study to assess the superiority of the SGLT2 inhibitor luseogliflozin over a DPP-4 inhibitor on ABPM results. Following enrolment and the provision of written informed consent (visit 1), the participants will undergo ABPM assessment twice using a TM 2433 device (A\&D Medical, Tokyo, Japan) to create a baseline. The ABPM device records $\mathrm{BP}$ and pulse rate every $30 \mathrm{~min}$ for 24 hours. At the same time, a log of the times of waking, going to bed and meal consumption will be kept. At each study visit, clinic BP, pulse rate, body mass and abdominal circumference will be measured. After this initial assessment, all the participants will be randomly assigned to continue their DPP-4 inhibitor or to switch to once daily luseogliflozin $(2.5 \mathrm{mg}$ / day) (visit 2). Serum and urine metabolic parameters will be assessed before and at the end of the study period. The randomisation and allocation of the participants will be performed by a specialised centre that is independent of the participating sites. After 8 weeks of treatment, ABPM and serum biomarker measurements will be performed again, as at baseline (visit 3) (figure 1).

\section{Recruitment strategy}

Participant enrolment will take place between 17 July 2018 and 31 March 2020 at five clinics located in Sapporo City. The inclusion and exclusion criteria for this trial are listed in boxes 1 and 2. Patients will be discontinued from the trial if any of the following criteria apply: (1)

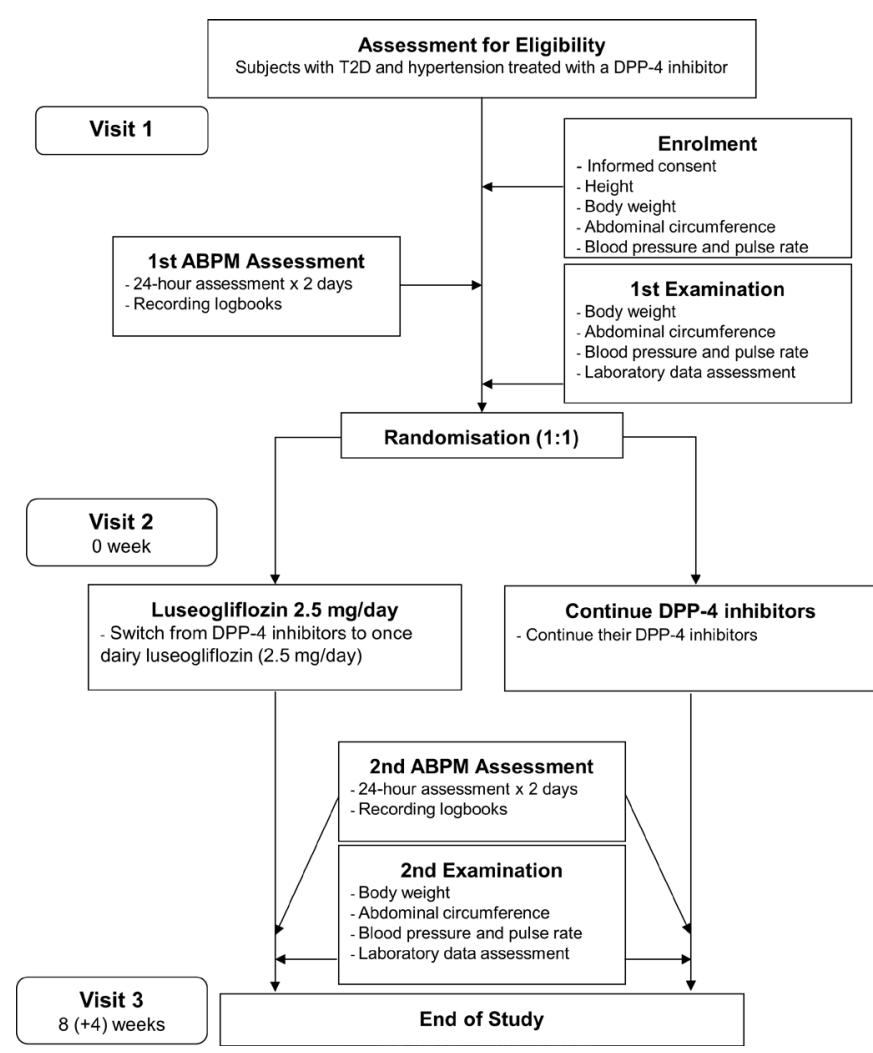

Figure 1 Patient recruitment scheme. Randomised participants will be assigned to continued DPP-4 inhibitor use or to switch to once-daily luseogliflozin $(2.5 \mathrm{mg} /$ day). All participants will undergo ABPM assessment twice at baseline and at the end of the study. ABPM, ambulatory blood pressure monitoring; DPP, dipeptidyl peptidase; T2D, type 2 diabetes.

withdrawal of consent, (2) physician's decision based on the patient's condition, (3) discontinuation of this clinical study or (4) physician's decision based on another reason.

\section{Intervention}

All the participants will be randomly assigned to continue their DPP-4 inhibitor or to switch to once daily luseogliflozin $(2.5 \mathrm{mg} /$ day $)$, and encouraged to continue their diet and exercise therapy during the study. The treatments will be supervised through the appropriate medical care centre for 8 weeks. The doses of anti-hypertensive drugs, anti-hyperglycemic agents, including DPP-4 inhibitors and luseogliflozin, will not be adjusted during the

\section{Box 1 Inclusion criteria}

- Japanese participants with T2D.

- Age $\geq 20$ and $<85$ years.

- $\mathrm{HbA1c} \geq 6.0 \%$ and $<9.0 \%$.

- Treatment with a DPP-4 inhibitor, and with the exception of onceweekly drugs, for at least for 4 weeks before enrolment.

- A diagnosis and exhibiting hypertension (BP $>130 / 80 \mathrm{~mm} \mathrm{Hg}$ ).

BP, blood pressure; DPP-4, dipeptidyl peptidase-4; HbA1c, haemoglobin A1c; T2D, type 2 diabetes. 


\section{Box 2 Exclusion criteria}

- Treatment with any SGLT2 inhibitor.

- An allergy against SGLT2 inhibitors.

- Unstable diabetic retinopathy.

- Current severe liver dysfunction or nephropathy.

Pregnancy.

- Severe ketosis.

- Diabetic coma or precoma.

- Severe infection, trauma and/or recent or planned surgery.

- Impairment in insulin secretion.

- $\mathrm{BMl}<22 \mathrm{~kg} / \mathrm{m}^{2}$.

- eGFR $<30 \mathrm{~mL} / \mathrm{min} / 1.73 \mathrm{~m}^{2}$.

- Incompatibility with an appropriate diet.

- Shift-working or irregular working hours.

- Incompatibility with the trial for other reasons, as determined by the physician.

BMI, body mass index; eGFR, estimated glomerular filtration rate; SGLT2, Sodium-glucose cotransporter 2

study period. The strategies to improve adherence to intervention protocol and procedures for monitoring adherence are as follows: (1) repeated instruction on the use of ABPM at each clinic visit; (2) when patients attend clinic visits with family members, the study and use of ABPM will be explained to both to the patient and his or her family member and (3) each clinic will designate dedicated staff to explain the study protocol to patients and their families.

\section{Consent to participate and withdrawal}

A clinician in the research team will obtain written informed consent from all eligible participants. The written material, consisting of a participant information leaflet and consent documentation, has been approved by the Research Committee. There will be an opportunity for the participants to ask questions freely to members of the research team, and their consent can be withheld at any time during the study period, should they wish.

\section{RANDOMISATION}

\section{Computer randomisation}

Randomisation is carried out centrally using a web-based system (Alloc System; https://www.croit.com/?s=alloc+ link\& $\mathrm{x}=0 \& \mathrm{y}=0)$. Given that ageing has been reported to significantly affect nocturnal hypertension ${ }^{12}$ and that the direct effects of hypoglycaemic actions and/or obesity should be minimised in this study, participants will be randomly assigned to continue their DPP-4 inhibitor or to switch to once-daily luseogliflozin $(2.5 \mathrm{mg} /$ day $)$, according to their age, BMI and HbA1c level.

\section{Treatment allocation}

All the participants will be randomly assigned to continue their DPP-4 inhibitor or to switch to once daily luseogliflozin $(2.5 \mathrm{mg} /$ day $)$.

\section{Study population}

Japanese participants with T2D aged $\geq 20$ and $<85$ years, HbA1c $\geq 6.0 \%$ and $<9.0 \%$, treatment with a DPP-4 inhibitor, and with the exception of once-weekly drugs, for at least for 4 weeks before enrolment, and a diagnosis of hypertension made prior to enrolment (see box 1).

\section{Patient and public involvement statement}

Participants were not directly involved in the design or development of the research and will not be involved in the recruitment and conduct of the study. Results of the examinations will be given to the participants after the study during a medical consultation in their participating centre.

\section{TRIAL ENDPOINT}

\section{Primary and secondary endpoints}

The primary endpoint of this study is the mean change in systolic blood pressure (SBP), measured by ABPM, during the night in participants with T2D. The secondary endpoints are as follows: the mean change in (1) diastolic blood pressure (DBP) during the night, (2) 24 hours of SBP and DBP, (3) daytime SBP and DBP, (4) pulse rate, (5) BP M-value, (6) trough SBP and DBP using the average ABPM reading for 1 hour before the next dose and (7) laboratory parameters indicative of glucose and lipid metabolism, and liver and renal function. The relationship between BP reduction and weight loss will also be assessed. 'Twenty-four hour' values are defined as the mean of all the readings obtained over a 24-hour period. In addition, we will determine whether participants in each group can be classified into three subcategories on the basis of their ABPM results before and after each treatment. The nocturnal SBP fall (\%) will be calculated as $100 \times$ [1-sleep SBP SBP/awake SBP ratio], and patients will be subclassified by nocturnal SBP fall as follows: 'nondippers', who show a nocturnal reduction in SBP of $<10 \%$ of the waking SBP; 'dippers', who show a nocturnal reduction of $\geq 10 \%$; and 'risers', who show a nocturnal increase of $\geq 0 \%{ }^{513}$

\section{Sample size and statistical analysis}

The sample size was calculated on the basis that luseogliflozin will improve nocturnal SBP by at least $10.7 \mathrm{~mm} \mathrm{Hg}$ (SD $11.3 \mathrm{~mm} \mathrm{Hg}$ ), as shown in a previous small-group single-arm pilot study of the use of luseogliflozin in Japanese T2D patients, evaluated using ABPM. ${ }^{14}$ Power calculations determined that a sample size of 25 individuals per group would be required to achieve a power of at least $90 \%$ for the detection of a difference between treatments. $\mathrm{p}<0.05$ will be considered to represent statistical significance and all tests will be two sided. Assuming five dropouts per each group, the sample size has been set at 30 participants per group. To ensure sufficient participant enrolment to achieve the target sample size, we will conduct the study at five clinics located in Sapporo City. We will also prepare an ABPM time-course sheet for each 
study visit to minimise the risk of patient discontinuation. We will perform ABPM twice before randomisation and after the treatment period, and the mean values obtained at each stage will be used for statistical analyses. For the primary endpoint, we will compare the mean change in nocturnal SBP between visit 1 and visit 3, after 8 weeks of luseogliflozin or DPP-4 inhibitor treatment.

Analysis of the primary and secondary endpoint data will be performed on the full analysis set (FAS), which will include subjects who are enrolled in this study and are assigned to treatment groups. Subjects who do not meet the inclusion criteria, for whom insufficient primary endpoint data are available, or who appreciably deviate from the study protocol will be excluded from the FAS. Differences between the two groups will be analysed for statistical significance using the unpaired t-test or MannWhitney U-test for continuous variables, and Pearson's $\chi^{2}$ test or the Fisher exact test for categorical data. We will analyse the data using JMP Pro V.14.1.1 (SAS Institute).

\section{ETHICS AND DISSEMINATION}

\section{Data protection and management}

Data management including coding, security, storage and cleaning will be performed by both researchers and an independent data management company (DOT WORLD Co., Ltd), whose employees will be contractually obliged to ensure that any personal information about potential and enrolled participants will be coded and handled confidentially before, during and after the trial. The study data will be archived at Hokkaido University for 5 years after study completion or 3 years after disclosure of the study results. Participants will be able to obtain the final results of the study. The UMIN and jRCT databases will contain detailed information on this study. Study conduct will be evaluated by a monitor independent from the investigators. No auditing of study sites is scheduled. According to the Clinical Trials Act in Japan, adverse events and other information including modification of the trial will be reported and disclosed publicly.

\section{DISCUSSION}

This clinical trial is designed to compare the differences in the anti-hypertensive effects of the SGLT2 inhibitor, luseogliflozin and DPP-4 inhibitors, especially at night, in patients with T2D and hypertension. The effective treatment of hypertension is often limited by the fact that most outpatients usually have their BP measured in the clinic or daily at home, and therefore their 24-hour BP profile is not usually available. BP measured in the clinic has been reported to be poorly representative of a patient's 24-hour BP profile, in contrast to ABPM. ${ }^{15}$ Furthermore, previous studies have shown that ABPM can provide evidence of early-stage macro- and microvascular complications in diabetic patients. ${ }^{16}$ Hence, nocturnal hypertension, identified using ABPM, represents an appropriate therapeutic target to reduce the incidence of such complications.
A previous phase 3 study assessing the efficacy of empagliflozin on 24-hour BP in patients with T2D (EMPA-REG BP) revealed that treatment with $10 \mathrm{mg}$ or $25 \mathrm{mg}$ of empagliflozin for 12 weeks significantly ameliorated both office BP and 24-hour BP, including nighttime BP. ${ }^{17}$ However, in the Japanese population, empagliflozin showed a more potent antihypertensive effect on nocturnal hypertension, although the effect was not significant compared with the control (placebo) group. ${ }^{18}$ In a clinical setting, it is important to know which antihyperglycemic agent is most effective at limiting cardiovascular mortality. Of the oral anti-hyperglycemic agents, DPP-4 inhibitors are the most frequently used anti-diabetic drugs in Japan. ${ }^{19}$ These agents ameliorate hyperglycemia in a glucose-dependent manner and are expected to have beneficial effects on atherosclerosis; however, large-scale clinical trials have so far failed to demonstrate a reduction in CVD risk. ${ }^{20-22}$ The strength of the present study is that it will be the first to directly compare the nocturnal anti-hypertensive efficacy of luseogliflozin and DPP-4 inhibitors. If luseogliflozin is shown to more effectively ameliorate nocturnal hypertension than DPP-4 inhibitors, there would be merit in switching from DPP-4 inhibitors to luseogliflozin, especially those with nocturnal hypertension. In addition, such a finding would be consistent with SGLT2 inhibitors having a protective effect against CVD, which has not been shown for DPP-4 inhibitors. ${ }^{7-9}$

Another advantage of using ABPM is that it can identify abnormal BP fluctuation, especially in the night time to early morning period. Normally, nocturnal BP decreases $>10 \%$ versus the daytime values (the 'dipper' pattern), but if this reduction is $<10 \%$ (the 'non-dipper' pattern) or if nocturnal BP is higher than during the daytime (the 'riser' pattern), higher CVD risk is associated. ${ }^{53}$ Therefore, we will categorise the participants according to these daily BP patterns and determine whether luseogliflozin is capable of normalising the abnormal patterns. Finally, it is worth mentioning that this study will be conducted in a standard clinical practice setting, which reflects real world.

In conclusion, this study will investigate whether luseogliflozin is more effective at ameliorating nocturnal hypertension, which has been shown to predispose to cardiovascular complications, than DPP-4 inhibitors. The result of this study may therefore provide new insights regarding the protective effects of SGLT2 inhibitors against CVD in patients with T2D.

\section{Author affiliations}

${ }^{1}$ Department of Rheumatology, Endocrinology and Nephrology, Faculty of Medicine and Graduate School of Medicine, Hokkaido University, Sapporo, Hokkaido, Japan

${ }^{2}$ Clinical Research and Medical Innovation Center, Hokkaido University Hospital, Sapporo, Hokkaido, Japan

${ }^{3}$ Sapporo Diabetes and Thyroid Clinic, Sapporo, Hokkaido, Japan

${ }^{4}$ Division of Diabetes and Endocrinology, Department of Medicine, Sapporo Medical Centre, NTT East Corporation, Sapporo, Japan

${ }^{5}$ Kurihara Clinic, Sapporo, Hokkaido, Japan

${ }^{6}$ Aoki Clinic, Sapporo, Hokkaido, Japan

${ }^{7}$ Division of Diabetes and Obesity, Faculty of Medicine and Graduate School of Medicine, Hokkaido University, Sapporo, Hokkaido, Japan 
Acknowledgements We thank Mark Cleasby, PhD, from Edanz Group (www. edanzediting.com/ac) for editing a draft of this manuscript.

Contributors RK, KYC and HM designed the original study protocol. HN, SK, KO, JT, SN, YK, SA, AN and TA contributed to modify the study design. RK and HN drafted the manuscript, and all the other authors contributed to its revision. All authors will contribute to participants enrollment. RK and KYC will collect the data and contribute to statistical analysis. HM is the guarantor of this work and will take responsibility for the integrity of the data and the accuracy of the data analysis.

Funding The study is funded by Taisho Pharmaceutical Co., Ltd, 3-24-1, Takada, Toshima-ku, Tokyo 170-8633 Japan. The data are owned by the investigators.

Competing interests AN has received honoraria for lectures from Sanofi, Mitsubishi Tanabe Pharma, Daiichi Sankyo, Eli Lilly Japan, MSD, Novo Nordisk Pharma, Novartis Pharma, AstraZeneca, Takeda Pharmaceutical, Astellas, Kowa Pharmaceutical, Ono, Nippon Boehringer Ingelheim, and Taisho Toyama Pharmaceutical, and has obtained research support from Mitsubishi Tanabe Pharma, Daiichi Sankyo, MSD, Novo Nordisk Pharma, Novartis Pharma, AstraZeneca, Dainippon Sumitomo Pharma, Life Scan Japan, and Taisho Pharmaceutical Co., Ltd. YK has received honoraria for lectures from Astellas Pharma Inc., AstraZeneca, Mitsubishi Tanabe Pharma Co., Ltd., MSD, Ono Pharmaceutical Co., Ltd., Sanofi, Shionogi \& Co., Ltd., Taisho Toyama Pharmaceutical Co., Ltd., and Takeda Pharmaceutical Co., Ltd. TA has received honoraria for lectures from Mitsubishi Tanabe Pharma, Chugai Pharmaceutical, Astellas Pharma, Takeda Pharmaceutical, Pfizer, and Eli Lilly, and has received research funding from Astellas Pharma, Takeda Pharmaceutical, Mitsubishi Tanabe Pharma, Chugai Pharmaceutical, Daiichi Sankyo, and Otsuka Pharmaceutical. HM has received honoraria for lectures from Astellas Pharma, AstraZeneca, Dainippon Pharma, Eli Lilly, Kissei, Mitsubishi Tanabe Pharma, MSD, Novo Nordisk Pharma, Novartis Pharma, and Sanofi, and has received research funding from Astellas Pharma, Astra-Zeneca, Eli Lilly, and Mitsubishi Tanabe Pharma.

Patient consent for publication Not required.

Ethics approval The study protocol has been approved by the Ethics Review Board of Hokkaido University Hospital (No. 017-0277), and the current version is 2.0 (approval on 7 February 2019). The study will be carried out according to the principles of Declaration of Helsinki and its amendments.

Provenance and peer review Not commissioned; externally peer reviewed.

Open access This is an open access article distributed in accordance with the Creative Commons Attribution Non Commercial (CC BY-NC 4.0) license, which permits others to distribute, remix, adapt, build upon this work non-commercially, and license their derivative works on different terms, provided the original work is properly cited, appropriate credit is given, any changes made indicated, and the use is non-commercial. See: http://creativecommons.org/licenses/by-nc/4.0/.

ORCID iD

Hideaki Miyoshi http://orcid.org/0000-0002-5909-3243

\section{REFERENCES}

1 Sarwar N, Gao P, Seshasai SRK, et al. Diabetes mellitus, fasting blood glucose concentration, and risk of vascular disease: a collaborative meta-analysis of 102 prospective studies.. Lancet 2010;375:2215-22.

2 UK Prospective Diabetes Study Group. Tight blood pressure control and risk of macrovascular and microvascular complications in type 2 diabetes: UKPDS 38. BMJ 1998;317:703-13.

3 Turnbull F, Neal B, Algert C, et al. Effects of different blood pressurelowering regimens on major cardiovascular events in individuals with and without diabetes mellitus: results of prospectively designed overviews of randomized trials. Arch Intern Med 2005;165:1410-9.

4 Ohkubo T, Hozawa A, Yamaguchi J, et al. Prognostic significance of the nocturnal decline in blood pressure in individuals with and without high 24-h blood pressure. J Hypertens 2002;20:2183-9.

5 Kario K, Pickering TG, Matsuo T, et al. Stroke prognosis and abnormal nocturnal blood pressure falls in older hypertensives. Hypertension 2001;38:852-7.

6 Mondick J, Riggs M, Sasaki T, et al. Mixed-Effects modelling to quantify the effect of empagliflozin on renal glucose reabsorption in patients with type 2 diabetes. Diabetes Obes Metab 2016;18:241-8.

7 Zinman B, Wanner C, Lachin JM, et al. Empagliflozin, cardiovascular outcomes, and mortality in type 2 diabetes. N Engl J Med 2015;373:2117-28.

8 Neal B, Perkovic V, Mahaffey KW, et al. Canagliflozin and cardiovascular and renal events in type 2 diabetes. N Engl J Med 2017;377:644-57.

9 Wiviott SD, Raz I, Bonaca MP, et al. Dapagliflozin and cardiovascular outcomes in type 2 diabetes. N Engl J Med 2019;380:347-57.

10 Baker WL, Smyth LR, Riche DM, et al. Effects of sodium-glucose cotransporter 2 inhibitors on blood pressure: a systematic review and meta-analysis. J Am Soc Hypertens 2014;8:262-75.

11 Zhang X, Zhao Q. Effects of dipeptidyl peptidase-4 inhibitors on blood pressure in patients with type 2 diabetes: a systematic review and meta-analysis. J Hypertens 2016;34:167-75.

12 Deng M, Chen D-W, Dong Y-F, et al. Independent association between age and circadian systolic blood pressure patterns in adults with hypertension. J Clin Hypertens 2017;19:948-55.

13 O'Brien E, Sheridan J, O'Malley K. Dippers and non-dippers. Lancet 1988;332:397.

14 Shirabe S, Maeda H, Koike Y, et al. Analysis of the effect of luseogliflozin on patients with type 2 diabetes and hypertension using ambulatory blood pressure monitoring. Journal of the Japan Diabetes Society 2017:60:S354

15 Hodgkinson J, Mant J, Martin U, et al. Relative effectiveness of clinic and home blood pressure monitoring compared with ambulatory blood pressure monitoring in diagnosis of hypertension: systematic review. BMJ 2011;342:d3621.

16 Jennersjö Pär E:son, Wijkman M, Wiréhn A-B, et al. Circadian blood pressure variation in patients with type 2 diabetes - relationship to macro- and microvascular subclinical organ damage. Prim Care Diabetes 2011:5:167-73.

17 Tikkanen I, Narko K, Zeller C, et al. Empagliflozin reduces blood pressure in patients with type 2 diabetes and hypertension. Diabetes Care 2015;38:420-8.

18 Kario K, Okada K, Kato M, et al. 24-Hour blood pressure-lowering effect of an SGLT-2 inhibitor in patients with diabetes and uncontrolled nocturnal hypertension: results from the randomized, placebo-controlled SACRA study. Circulation 2018. doi: 10.1161/ CIRCULATIONAHA.118.037076. [Epub ahead of print: 29 Nov 2018].

19 Kubota K, Kamijima Y, Kao Yang Y-H, et al. Penetration of new antidiabetic medications in East Asian countries and the United States: a cross-national comparative study. PLoS One 2018;13:e0208796.

20 Scirica BM, Bhatt DL, Braunwald E, et al. Saxagliptin and cardiovascular outcomes in patients with type 2 diabetes mellitus. $N$ Engl J Med 2013;369:1317-26.

21 White WB, Cannon CP, Heller SR, et al. Alogliptin after acute coronary syndrome in patients with type 2 diabetes. N Engl $\mathrm{J} \mathrm{Med}$ 2013;369:1327-35

22 Green JB, Bethel MA, Armstrong PW, et al. Effect of sitagliptin on cardiovascular outcomes in type 2 diabetes. N Engl J Med 2015;373:232-42.

23 Gökdeniz T, Kalaycıoğlu E, Aykan Ahmet Çağrı, et al. Effects of nondipper blood pressure pattern on left ventricular rotational mechanics in hypertensive patients with type 2 diabetes mellitus: a speckle tracking study. Int J Cardiovasc Imaging 2014;30:57-65. 